\title{
Preparation and characterization of genipin-cross-linked chitosan microparticles by water-in-oil emulsion solvent diffusion method
}

\author{
Jesada Karnchanajindanun, Mangkorn Srisa-ard, Prasong Srihanam, Yodthong Baimark* \\ Department of Chemistry and Center of Excellence for Innovation in Chemistry, Faculty of Science, Mahasarakham University, \\ Mahasarakham, Thailand; *Corresponding Author: yodthong.b@msu.ac.th.
}

Received 27 July 2010; revised 30 August 2010; accepted 5 September 2010.

\begin{abstract}
Chitosan (CS) microparticles with and without cross-linking were prepared by a water-in-oil emulsion solvent diffusion method without any surfactants. Aqueous CS solution and ethyl acetate were used as water and oil phases, respectively. Genipin was used as a cross-linker. Influences of genipin ratios and cross-linking times on CS microparticle characteristics were investigated. Non-cross-linked and cross-linked CS microparticles were spherical in shape and rough in surface. Microparticle matrices showed porous structures. Surface roughness, mean particle sizes and bulk density of CS microparticles increased and their dissolutions in acetic acid solution decreased when genipin ratio and crosslinking time increased.
\end{abstract}

Keywords: Chitosan microparticles; Porous structure; Genipin; Cross-linking; Morphology

\section{INTRODUCTION}

Chitosan is a copolymer of 2-glucosamine and $\mathrm{N}$ acetyl-2-glucosamine prepared by alkaline deacetylation of chitin that has received great attention for its possible uses in medical, pharmaceutical and metal ion treatment applications because of its biodegradability, biocompatibility and high concentration of amine functional groups [1-4]. Chitosan microparticles have usually been fabricated by precipitation, spray drying and water-in-oil (W / O) emulsification-cross-linking methods [5]. The current study describes an alternative method for preparation of cross-linked and non-cross-linked CS microparticles by the $\mathrm{W} / \mathrm{O}$ emulsion solvent diffusion method.

Genipin was selected as a biocompatible cross-linker. Genipin is a natural water-soluble bi-functional crosslinker. It is obtained from geniposide, a component of traditional Chinese medicine and is isolated from the fruits of the plant, Gardenial jasminoides Ellis [6]. Genipin is a fully biocompatible reagent about 10,000 times less cytotoxic than glutaraldehyde [7]. The CS device matrices were successfully cross-linked with genipin [8-10].

In this work, a novel approach to the preparation of genipin-cross-linked chitosan microparticles by the simple $\mathrm{W} / \mathrm{O}$ emulsion solvent diffusion method is reported. Cross-linked chitosan microparticles were solidified and formed after diffusion out of water from emulsion droplets of chiosan solution to external continuous phase, ethyl acetate. The influences of cross-linker ratio and cross-linking time on CS microparticle characteristics including morphology, particle size, dissolution and bulk density were investigated and discussed.

\section{EXPERIMENTAL}

\subsection{Materials}

Chitosan with degree of de-acetylation and average molecular weight of $90 \%$ and $100 \mathrm{kDa}$, respectively was purchased from Seafresh Chitosan Lab Co., Ltd. (Thailand). Genipin (Challenge Bioproducts Co. Ltd., Taiwan) and analytical grade ethyl acetate (Lab Scan) were used without further purification.

\subsection{Preparation of Chitosan Microparticles}

Chitosan solution with $1 \% \mathrm{w} / \mathrm{v}$ was prepared by using a $2 \%(\mathrm{v} / \mathrm{v})$ acetic acid aqueous solution as a solvent. Chitosan microparticles were prepared by the waterin-oil emulsion solvent diffusion method. The $0.5 \mathrm{~mL}$ of $0.5 \% \mathrm{w} / \mathrm{v}$ chitosan solution was added drop-wise to 200 $\mathrm{mL}$ of ethyl acetate with a stirring speed of $900 \mathrm{rpm}$ for $1 \mathrm{~h}$. The beaker was tightly sealed with aluminum foil during the emulsification-diffusion process to prevent ethyl acetate evaporation. The chitosan microparticles suspended in ethyl acetate were collected by centrifuga- 
tion before drying in a vacuum oven at room temperature for $6 \mathrm{~h}$.

Genipin-cross-linked chitosan microparticles were produced by the same method. The chitosan and genipin solutions were mixed together under constant stirring at room temperature for cross-linking before microparticle preparation. The final chitosan concentration was $0.5 \%$ $\mathrm{w} / \mathrm{v}$ after cross-linking. The different genipin concentrations $(5 \%, 10 \%$ and $20 \% \mathrm{w} / \mathrm{w})$ and cross-linking times (1.5, 3 and $6 \mathrm{~h})$ were investigated.

\subsection{Characterization of Chitosan Microparticles}

Morphology of the chitosan microparticles was determined by scanning electron microscopy (SEM) using a JEOL JSM-6460LV SEM. The microparticles were coated with gold for enhancing conductivity before scan. Mean microparicle size and the coefficient of variation (CV) were calculated for each on SEM images by counting a minimum of 100 particles using smile view software (version 1.02). The $\mathrm{CV}$ value was calculated from the following equation:

$$
\mathrm{CV}=\frac{\sigma}{D_{p}} \times 100
$$

where $\sigma$ is a standard deviation, and $D_{p}$ is a mean microparticle diameter measured from SEM images. Lower CV values indicate high microparticle monodispersity in size.

Percentage of dissolution of the chitosan microparticles was investigated by shaking $50 \mathrm{mg}$ of the chitosan microparticles in $1.5 \mathrm{~mL}$ of $2 \% \mathrm{w} / \mathrm{v}$ acetic acid aqueous solution at room temperature for $24 \mathrm{~h}$. The residue microparticles were recovered by centrifugation before drying in a vacuum oven at $50^{\circ} \mathrm{C}$ until its weight remained constant. The percentage of dissolution of the chitosan microparticles was then calculated by following the equation. Each average percentage of dissolution value was calculated from a mean of three measurements (see Eq.2).

where initial and remaining CS microparticles are the weights of chitosan microparticles before and after dissolution test, respectively.

Bulk density of the chitosan microparticles was measured by gas displacement method using an ultrapycnometer 1000 (Quantachrom, USA) under helium gas. Each average density value was calculated from a mean of five determinations.

\section{RESULTS AND DISCUSSIONS}

Here, the water-in-oil (W / O) emulsion solvent diffu- sion method without any surfactant was used to prepare chitosan (CS) microparticles. The maximum water solubility in ethyl acetate is $3.30 \%$ (CAS No. 141-78-6). Then the polymer particles should form, if the less than 3.3 $\mathrm{mL}$ of aqueous polymer solution ( $\mathrm{W}$ phase) was added drop-wise into $100 \mathrm{~mL}$ of ethyl acetate (O phase) with stirring. The water in dispersed emulsion droplets of CS solution diffused out to the continuous phase, ethyl acetate. It was found in our cases that the CS microparticles were successfully prepared using this method. In our preliminary test, the magnetic stirring speed of $900 \mathrm{rpm}$ is found the most appropriate for microparticle preparation. The almost aqueous CS solution could not be broken to form uniform droplets when the stirring speed was lower than $900 \mathrm{rpm}$. Meanwhile, almost all particles were stuck at the wall of glassware during emulsification-diffusion process when higher stirring speed than $900 \mathrm{rpm}$ was applied. The chitosan microparticles could not be completely solidified when the stirring time was shorter than $1 \mathrm{~h}$. The CS aggregates were found when higher $0.5 \mathrm{~mL}$ of $\mathrm{CS}$ solution was used due to its high viscosity of CS solution.

\subsection{Morphology and Sizes of CS Microparticles}

Figure 1 shows SEM images of non-cross-linked and cross-linked CS microparticles prepared with different genipin ratios. They were nearly spherical in shape suggested that the genipin ratios did not effect on the particle shape. Surfaces of these CS microparticles are illustrated in Figure 2. The non-cross-linked CS microparticles in Figure 2(a) showed rough surfaces. This may occur from diffusion out of water from dispersed droplets of CS solution to continuous ethyl acetate phase during particle solidification. The surface roughness increased with the genipin ratio as shown in Figures 2(b) -2(d). The results may be explained that the cross- linking can increase viscosity of CS solution. The diffusion out of water from cross-linked CS solution droplets was difficult.

The cross-linked CS microparticles prepared with different cross-linking times also showed the spherical-like shape, as illustrated in Figures 3(b)-3(d). This indicates that cross-linking times did not effect on the particle shape. The CS microparticles prepared with longer crosslinking time showed rougher surface than the shorter cross-linking time, as shown in Figure 4. This may be due to increasing of the viscosity of CS solution when the cross-linking time increased.

$$
\% \text { dissolution }=\frac{[\text { initial CS microparticles }(\mathrm{mg})-\text { remaining CS microparticles }(\mathrm{mg})]}{\text { initial CS microparticles }(\mathrm{mg})} \times 100
$$



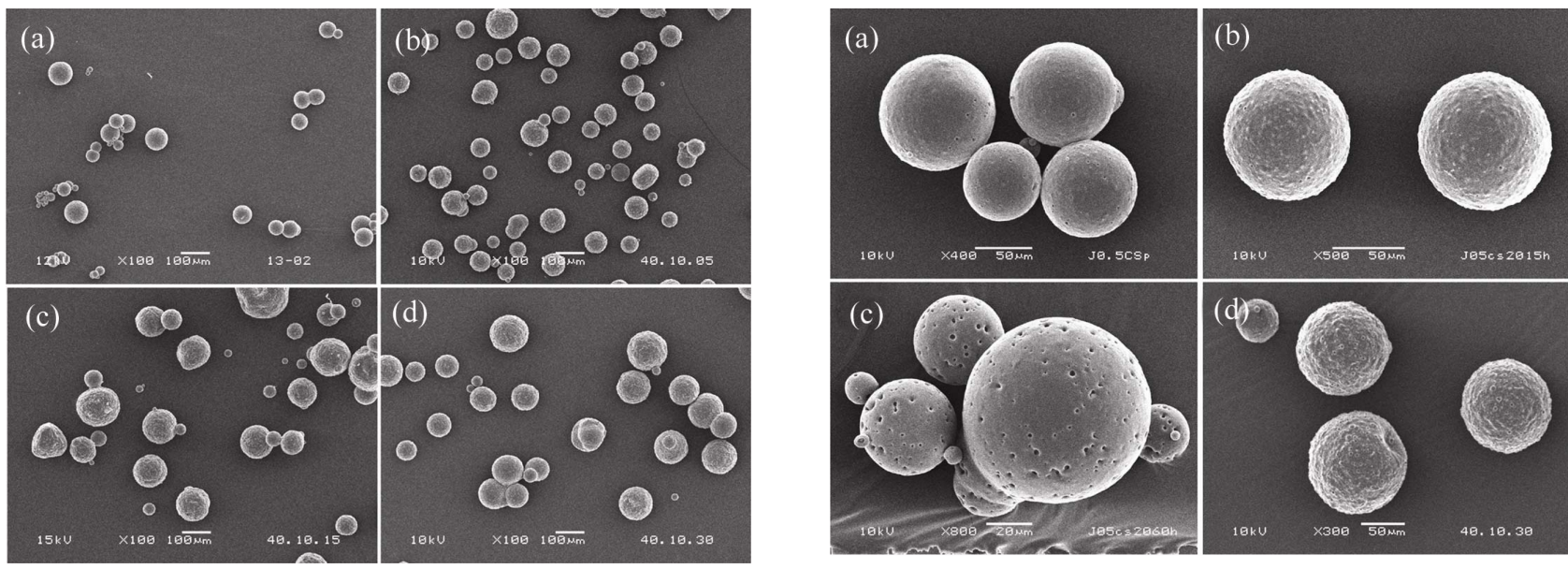

Figure 1. SEM micrographs of (a) non-cross-linked chitosan microparticles and cross-linked chitosan microparticles with genipin ratios of (b) $5 \%$, (c) $10 \%$ and (d) $20 \% \mathrm{w} / \mathrm{w}$ for cross-linking time of $6 \mathrm{~h}$. All bars $=100 \mu \mathrm{m}$.

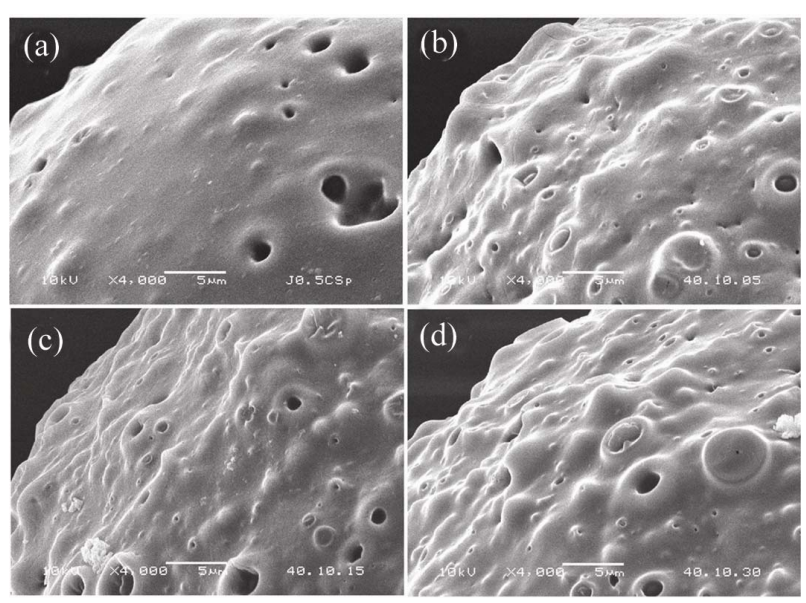

Figure 2. Expanded SEM micrographs of surfaces of (a) noncross-linked chitosan microparticles and cross-linked chitosan microparticles with genipin ratios of (b) $5 \%$, (c) $10 \%$ and (d) $20 \% \mathrm{w} / \mathrm{w}$ for cross-linking time of $6 \mathrm{~h}$. All bars $=5 \mu \mathrm{m}$.

Internal morphology of the CS microparticle matrix was examined through their broken surfaces (Figure 5). It can be seen that the microparticle matrices contained porous structure, resembling a sponge. These sponge-like particles were presumably created by rapid solidification of the chitosan matrix during diffusion out of the water from emulsion droplets. However, the porous structures were completely covered with a continuous outer particle surface. The matrix of cross-linked CS micro-particles was denser than that of the non-cross-linked microparticles. As example of which is shown in Figure 5(b) for the cross-linked CS microparticles prepared with $20 \%$ w/w genipin ratio compared with the non-cross-linked CS microparticles in Figure 5(a).

Figure 3. SEM micrographs of (a) non-cross-linked chitosan microparticles and cross-linked chitosan microparticles with cross-linking times of (b) 1.5, (c) 3 and (d) $6 \mathrm{~h}$ for genipin ratio of $20 \% \mathrm{w} / \mathrm{w}$. All bars $=50 \mu \mathrm{m}$.

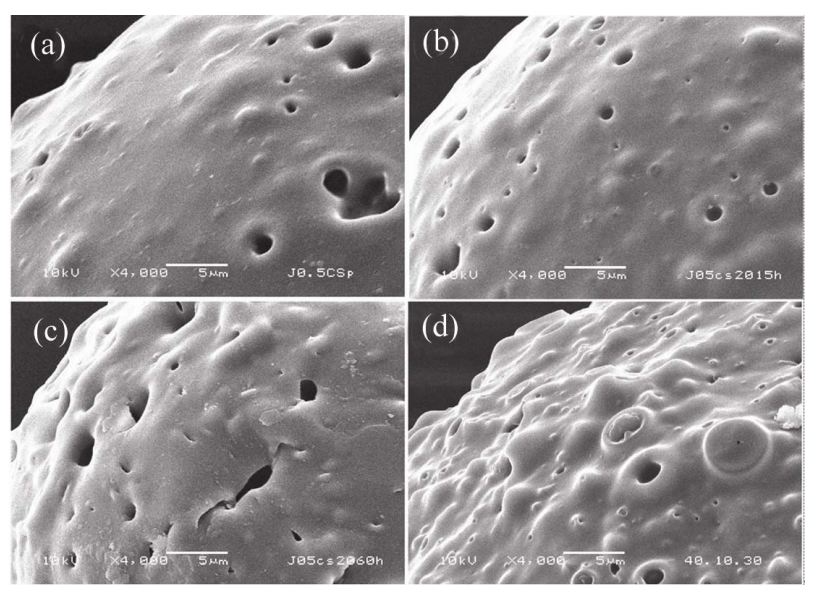

Figure 4. Expanded SEM micrographs of surfaces of (a) noncross-linked chitosan microparticles and cross-linked chitosan microparticles with cross-linking times of (b) 1.5, (c) 3 and (d) $6 \mathrm{~h}$ for genipin ratio of $20 \% \mathrm{w} / \mathrm{w}$. All bars $=5 \mu \mathrm{m}$.

Mean particle sizes of the CS microparticles were determined from several SEM images instead of scattering method (suspension in water) because of the partial swelling and dissolution of microparticles. The mean particle sizes and CV of the CS microparticles are summarized in Table 1. It was found that the particle sizes increased when the genipin ratio and the cross-linking time were increased. The CV for every sample is below $30 \%$ indicated the CS microparticles with low dispersity in size were formed. The results suggest that the CV of microparticles did not appear to affect the genipin ratio and cross-linking time.

\subsection{Dissolution of CS Microparticles}

Dissolution behavior of the CS microparticles indi- 


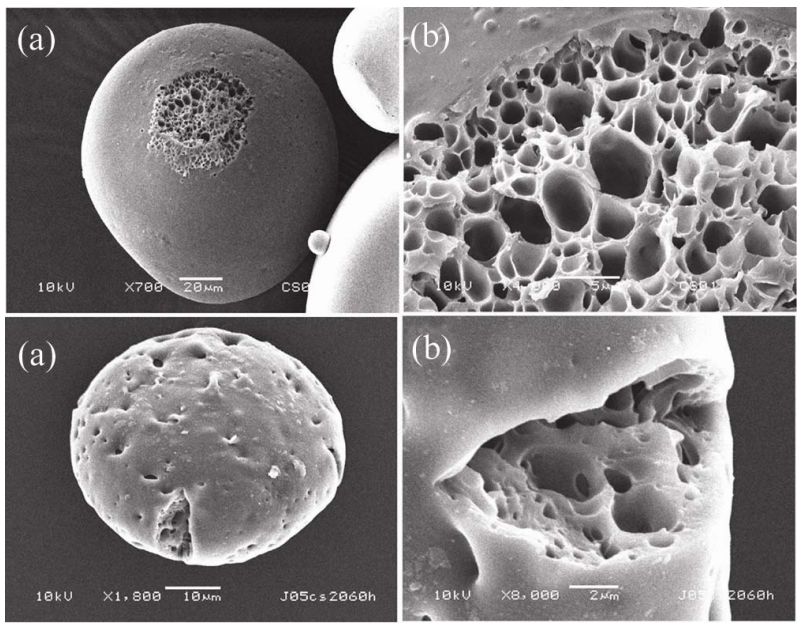

Figure 5. Low-magnification (left column) and high-magnification (right column) SEM micrographs of broken surfaces of (a) non-cross-linked chitosan microparticles and (b) cross-linked chitosan microparticles with $20 \% \mathrm{w} / \mathrm{w}$ genipin ratio for crosslinking time of $6 \mathrm{~h}$. Bars $=20$ and $10 \mu \mathrm{m}$ for (a) and (b), respectively in left column. Bars $=5$ and $2 \mu \mathrm{m}$ for (a) and (b), respectively in right column. rectly related to the degree of cross-linking. The higher dissolution of CS microparticles related to lower degree of cross-linking. Figure 6 shows dissolution of the CS microparticles in acetic acid solution for $24 \mathrm{~h}$. The noncross-linked CS microparticles were completely dissolved. This due to the weak acid solution such as acetic acid solution is a good solvent for CS. The dissolution of $\mathrm{mi}$ croparticles was decreased when the CS was cross-linked and increasing the genipin ratio and cross-linking time. The results suggest that the degree of cross-linking increased as the genipin ratio and cross-linking time increased, according to the literatures [8,9]. This is an important advantage for application in drug delivery with controllable drug release rate. Thus the drug release rates can be tailored by varying the degree of cross-linking.

\subsection{Bulk Density of CS Microparticles}

The porous structures of CS microparticel matrices with and without cross-linking can be clearly determined from their density values, as shown in Figure 7. It was found that the density values increased with the genipin

Table 1. Conditions for preparing chitosan microparticles and their particle sizes.

\begin{tabular}{ccccccc}
\hline & \multicolumn{7}{c}{ Sample No. } \\
\cline { 2 - 7 } Process parameter & 1 & 2 & 3 & 4 & 5 & 6 \\
\hline Genipin ratio (\%w/w) & 0 & 5 & 10 & 20 & 20 & 20 \\
Cross-linking time (h) & 0 & 6 & 6 & 6 & 1.5 & 3 \\
Mean particle size $(\mu \mathrm{m})$ & 85 & 98 & 110 & 112 & 92 & 104 \\
CV $(\%)$ & $24 \%$ & $27 \%$ & $25 \%$ & $29 \%$ & $28 \%$ & $29 \%$ \\
\hline
\end{tabular}
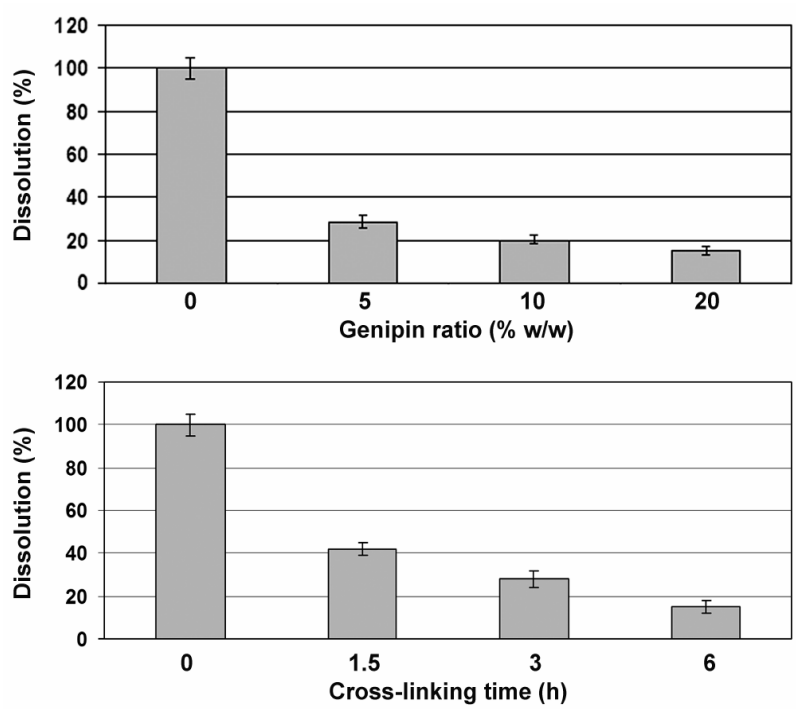

Figure 6. Dissolution of chitosan microparticles in $2 \% \mathrm{w} / \mathrm{v}$ acetic acid solution for $24 \mathrm{~h}$ prepared with different genipin ratios for cross-linking time of $6 \mathrm{~h}$ (above) and different crosslinking times for genipin ratio of $20 \% \mathrm{w} / \mathrm{w}$ (bottom).
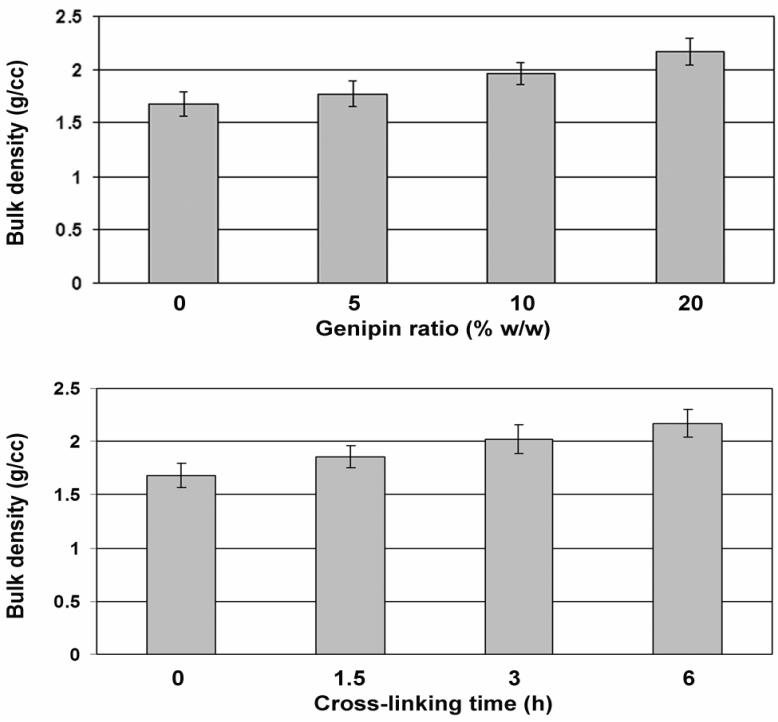

Figure 7. Bulk density of chitosan microparticles prepared with different genipin ratios for cross-linking time of $6 \mathrm{~h}$ (above) and different cross-linking times for genipin ratio of $20 \% \mathrm{w} / \mathrm{w}$ (bottom). 
ratio and cross-linking time. This can be explained that the CS molecules were closer together when the higher genipin ratio and cross-linking time were used. Therefore, the denser microparticles were obtained. The bulk density change after cross-linking corresponded to the microparticle matrices of the broken CS microparticles from the SEM images in Figure 5.

\section{CONCLUSIONS}

Non-cross-linked and genipin-cross-linked chitosan microparticles with spherical-like shapes have been successfully prepared using the simple and rapid $\mathrm{W} / \mathrm{O}$ emulsion solvent diffusion method. The surface roughness of microparticles increased with genipin ratio and crosslinking time but the particle shape did not change. All chitosan microparticle matricess contained porous structures. The cross-linked microparticles showed denser matrices than that of non-cross-linked microparticles. The mean particle sizes and bulk density of microparticles slightly increased as increasing the genipin ratio and the cross-linking time.

This simple $\mathrm{W} / \mathrm{O}$ emulsion solvent diffusion method is promising for the preparation of drug-loaded chitosan microparticles with and without cross-linking, especially water-soluble drugs. Drug release rates from microparticles might be controlled by adjusting the genipin ratio and/or cross-linking time.

\section{ACKNOWLEDGEMENTS}

This work was supported by Mahasarakham University (fiscal year 2011), the National Metal and Materials Technology Center (MTEC), National Science and Technology Development Agency (NSTDA), Ministry of Science and Technology, Thailand (MT-B-52-BMD68-180-G) and the Center of Excellence for Innovation in Chemistry (PERCH-CIC), Commission on Higher Education, Ministry of Education, Thailand.

\section{REFERENCES}

[1] Kumar, M.N.V.R., Muzzarelli, R.A.A., Muzzarelli, C., Sashiwa, H. and Domb, A.J. (2004) Chitosan chemistry and pharmaceutical perspectives. Chemical Review, 104, 6017-6084.

[2] Muzzarelli, R.A.A. and Muzzarelli, C. (2005) Chitosan chemistry: Relevance to the biomedical sciences. Advances in Polymer Science, 186, 151-209.

[3] Crini, G. (2005) Recent developments in polysaccharidebased materials used as adsorbents in wastewater treatment. Progress in Polymer Science, 30, 38-70.

[4] Learoyd, T.P., Burrows, J.L., French, E. and Seville, P.C. (2008) Modified release of beclometasone dipropionate from chitosan-based spray-dried respirable powders. Powder Technology, 187, 231-238.

[5] Agnihotri, S.A., Mallikarjuna, N.N. and Aminabhavi, T. M. (2004) Recent advances on chitosan-based micro- and nanoparticles in drug delivery. Journal of Controlled Release, 100, 5-28.

[6] Mi, F.L., Shyu, S.S. and Peng, C.K. (2005) Characterization of ring-opening Polymerization of genipin and $\mathrm{pH}-$ dependent cross-linking reactions between chitosan and genipin, Journal of Polymer Science, Part A: Polymer Chemistry, 43, 1985-2000.

[7] Nishi, C., Nakajima, N. and Ikada, Y. (1995) In vitro evaluation of cytotoxicity of diepoxy compounds used for biomaterial modification. Journal of Biomedical Material Research, 29, 829-834.

[8] Yuan, Y., Chesnutt, B.M., Utturkarr, G., Haggard, W.O., Yang, Y., Ong, J.L. and Bumgardner, J.D. (2007) The effect of cross-linking of chitosan microspheres with genipin on protein release. Carbohydrate Polymers, 68, 561567.

[9] Silva, S.S., Motta, A., Rodrigues, M.T., Pinheiro, A.F.M., Gomes, M.E., Mano, J.F., Reis, R.L. and Migliaresi, C. (2008) Novel genipin-cross-linked chitosan/silk fibroin sponges for cartilage engineering strategies. Biomacromolecules, 9, 2764-2774.

[10] Muzzarelli, R.A.A. (2009) Genipin-crosslinked chitosan hydrogels as biomedical and pharmaceutical aids. Carbohydrate Polymers, 77, 1-9. 